\title{
La presencia de obsidiana en contextos arqueológicos del norte de Mendoza: interacciones humanas en una región andina sin fuentes volcánicas
}

\author{
Valeria Cortegoso, Lucía Yebra, Silvina Castro y Víctor Durán \\ Recibido 12 de febrero 2019. Aceptado 14 de mayo 2019
}

\begin{abstract}
RESUMEN
Se presentan resultados del análisis geoquímico (XRF) de 61 piezas de obsidiana correspondientes a diez sitios arqueológicos del norte de Mendoza $\left(32^{\circ}-33^{\circ} \mathrm{S}\right)$; un segmento latitudinal donde la cordillera de los Andes alcanza su máxima altitud. Además de estar fuera de la región andina con fuentes de obsidiana, los sitios se localizan al norte del límite de divergencia cultural establecida en los últimos milenios $\left(34^{\circ} \mathrm{S}\right)$ entre sociedades productoras y cazadoras-recolectoras. Se evalúa la asignación de elementos de obsidiana a fuentes cordilleranas y extracordilleranas entre $\left(34^{\circ}-37^{\circ} \mathrm{S}\right)$ todas en un rango comprendido entre 200 y $500 \mathrm{~km}$ de distancia. Las cronologías de los contextos abarcan un extenso rango temporal (entre ca. 7400 y 300 años AP) durante el cual se registra un proceso de diversificación económica que separa a las sociedades del área considerada de sus vecinas ubicadas al sur. Los resultados contribuyen a evaluar la dirección de interacciones que involucran principalmente a la cordillera, pero además discutir cambios en escala y modalidad de acceso a las fuentes entre grupos con distintas estrategias económicas.
\end{abstract}

Palabras clave: Obsidianas arqueológicas; Geoquímica; Norte de Mendoza; Interacciones humanas.

\begin{abstract}
ARCHAEOLOGICAL OBSIDIAN IN NORTHERN MENDOZA: HUMAN INTERACTIONS IN AN ANDEAN REGION THAT LACKS VOLCANIC SOURCES. The results of geochemical analysis (XRF) of obsidian artifacts $(n=61)$ belonging to ten archaeological sites in northern Mendoza $\left(32^{\circ}-33^{\circ} \mathrm{S}\right)$ are presented. The sites are located outside of the Andean volcanic sources and to the north of the frontier $\left(34^{\circ}\right)$ established in recent millennia between farmers and hunter-gatherer societies. The assignation of obsidian artifacts to cordilleran and extra cordilleran sources $\left(34^{\circ}\right.$ y $\left.37^{\circ} \mathrm{S}\right)$ is evaluated, all of which are located between 200 and $500 \mathrm{~km}$ from the study area. Archaeological contexts correspond to a chronological range of c.7400 to 300 years $\mathrm{BP}$, during which period processes of economic diversification have been recorded that separated societies within the study area from their southern neighbors. The results contribute to the assessment of human interactions along the Andean cordillera, but also suggest changes in scale and modalities of access to sources among groups with different economic strategies.
\end{abstract}

Keywords: Archaeological obsidians; Geochemistry; North of Mendoza; Human interactions.

Valeria Cortegoso. Consejo Nacional de Investigaciones Científicas y Técnicas (CONICET), Instituto Interdisciplinario
de Ciencias Básicas (ICB), Universidad Nacional de Cuyo (UNCuyo). Padre Jorge Contreras I 300 (5500), Mendoza,
Argentina. E-mail: vcortegoso@gmail.com
Lucía Yebra. CONICET, ICB, UNCuyo. Padre Jorge Contreras I 300 (5500), Mendoza, Argentina. E-mail: yebralucia@hotmail.com
Silvina Castro. Facultad de Filosofía y Letras, UNCuyo. Padre Jorge Contreras I 300 (5500), Mendoza, Argentina. E-mail:
silvinacastro20 I5@gmail.com
Víctor Durán. CONICET, ICB, UNCuyo. Padre Jorge Contreras I300 (5500), Mendoza, Argentina. E-mail: duranvic2@gmail.com

Intersecciones en Antropología 20 (2), agosto-diciembre: 153-165. 2019. ISSN-e 1850-373X

Facultad de Ciencias Sociales - UNICEN - Argentina 


\section{INTRODUCCIÓN}

La cordillera de los Andes es el rasgo topográfico más significativo de Sudamérica y en gran medida modela las características ambientales del continente. Su incidencia como barrera en la circulación atmosférica condiciona los regímenes de precipitación y la estructura fitogeográfica en ambas vertientes (Garreaud et al. 2008). En este trabajo enfocamos el segmento latitudinal, donde la cordiIlera alcanza su mayor altitud $\left(32^{\circ} \mathrm{S}\right)$, con picos por encima de los $6000 \mathrm{msnm}$ y pasos intercordilleranos a casi $4000 \mathrm{msnm}$. El relieve, la altitud y la segmentación estacional en la oferta de recursos impactan en las estrategias humanas. Estos rasgos biogeográficos explican, entre otros aspectos, el desarrollo de sociedades diversas en ambas vertientes cordilleranas, como así también una extendida historia de interacciones que las conectaron (Lagiglia 1979). ${ }^{1}$

Este trabajo se enmarca en una investigación que enfoca desde una perspectiva comparativa el análisis de la dinámica demográfica e interacción interpoblacional en distintas franjas latitudinales de la cordillera de los Andes entre $32^{\circ}-36^{\circ} \mathrm{S} .^{2}$ Estudios previos las identifican como áreas con contrastes marcados en cuanto a condiciones para el acceso y circulación desde ambas vertientes, lo que permitió plantear la existencia de vectores geográficos dominantes de circulación transcordillerana (Cortegoso et al. 2016; Barberena et al. 2017). Se ha indagado sobre fenómenos que condujeron durante el Holoceno tardío al desarrollo cultural divergente al norte y sur de los $34^{\circ}$ (Cortegoso et al. 2014). ${ }^{3}$ Uno de los ejes es el estudio de procedencia de obsidianas; un progresivo relevamiento y caracterización han permitido identificar nueve señales químicas correspondientes a fuentes con variable extensión y calidad. El análisis geoquímico de más de 3000 artefactos arqueológicos de Argentina y Chile ha permitido discutir la variabilidad espacial y temporal respecto de su explotación y circulación a escalas extrarregionales. Se ha evaluado la asimetría de la distribución arqueológica en ambas vertientes andinas como un modo de identificar las áreas desde las que se accedió a las fuentes y las regiones que alcanzaron los artefactos por medio del acceso indirecto (Durán et al. 2018).

El norte de Mendoza está emplazado fuera del área de actividad volcánica y carece de fuentes de obsidiana; es un recurso minoritario en los contextos arqueológicos $(<0,5 \%)$. En áreas montañosas entre $32^{\circ}$ y $33^{\circ} \mathrm{S}$ se han estudiado fuentes de otras materias primas, principalmente de rocas silíceas criptocristalinas, riolitas y basaltos (Cortegoso 2008; Cortegoso et al. 2017), que forman la mayor parte del registro lítico.

Se discuten los resultados del análisis geoquímico de $\mathrm{N}=61$ piezas de obsidiana procedentes de diez sitios arqueológicos localizados entre $32^{\circ}$ $28^{\prime} 51.67^{\prime \prime} \mathrm{S}$ y $33^{\circ} 40^{\prime} 0.65^{\prime \prime} \mathrm{S}$. Se trata en su totalidad de materias primas alóctonas (Meltzer 1989; Civalero y Franco 2003; Stern 2018), ya que todos los artefactos de obsidiana provienen de distintas áreas cordilleranas limítrofes o extracordilleranas de la vertiente oriental emplazadas en un rango de entre 200 y $500 \mathrm{~km}$ de distancia.

Las cronologías de los contextos abarcan un rango temporal amplio que incluye el Holoceno medio inicial y principalmente el tardío (ca. 7400 años AP y 300 años AP). La asignación de elementos de obsidiana correspondientes a distintos contextos en un registro que incluye el cambio de estrategias humanas contribuye a evaluar interacciones que involucran a la cordillera. Permite además discutir cambios en escala y modalidad de acceso a las fuentes desde sociedades de cazadores y recolectores a grupos productores más tardíos.

Los estudios fueron realizados con equipos portátiles de XRF de la Universidad de Missouri (MURR) y las asignaciones han sido determinadas por el Dr. Michael Glascock. En base a estos resultados se discuten alternativas sobre vías de circulación y abastecimiento de bienes exóticos. También se evalúan los rasgos tecnológicos que muestra este recurso alóctono en las distintas unidades geomorfológicas y segmentos temporales.

\section{MARCO REGIONAL Y ANTECEDENTES}

Los Andes se elevaron como consecuencia de la subducción de varias placas oceánicas por debajo de la continental, que dio origen a uno de los arcos magmáticos más extendidos del mundo, que forma parte del cinturón de fuego que bordea al océano Pacífico. El volcán Tupungato $\left(33^{\circ} \mathrm{S}\right)$ marca la finalización del arco volcánico cuaternario debido al cambio del ángulo de inclinación de la placa subducida, que hacia el norte tiende a ser subhorizontal (Llambías et al. 2010). Esto explica la ausencia de fuentes de obsidiana en el norte de Mendoza. A partir de $28^{\circ} \mathrm{S}$ se inicia nuevamente un área cordillerana donde distintos tipos de actividades 
volcánicas generaron las condiciones para la existencia de fuentes de obsidiana (Yacobaccio et al. 2004).

El programa de relevamiento en la región de estudio ha permitido identificar señales químicas correspondientes a fuentes primarias y secundarias y canteras-taller con una representación significativa en el registro arqueológico regional. Cuatro fuentes son cordilleranas: Laguna del Maule 1 (LM1), Las Cargas (LC), Arroyo Paramillos (AP) y Laguna del Diamante (LD); y tres son extracordilleranas: Cerro Huenul (HU), El Peceño (EP) y Maule 2 (LM2) (Figura 1) (Seelenfreund et al. 1996; Durán et al. 2004; De Francesco et al. 2006; Giesso et al. 2011; Barberena et al. 2018). Además, se han identificado dos tipos químicos recurrentes en muestras arqueológicas de Argentina y Chile, denominados Desconocidas A y B (DESC-A y DESC-B), sin referencia geográfica sobre las fuentes de origen.

Todas las fuentes cordilleranas se encuentran en ambientes limítrofes a más de 2500 msnm, por lo que el acceso a ellas es estacional. Su uso mayoritario en toda la secuencia humana de explotación en la región es un patrón que surge de los antecedentes mencionados. Las fuentes LC y LM son las más extensas, con la mayor distribución en el sur de Mendoza, y registran una explotación temprana y con ritmo creciente hasta el contacto hispanoindígena (Cortegoso et al. 2012; Durán et al. 2012; Salgán et al. 2015). ${ }^{4}$ Las fuentes AP y LD tienen un patrón de uso distinto, con una concentración en el área cordillerana y distribución mayoritaria hacia la cuenca del Maipo, en la vertiente occidental andina (Cortegoso et al. 2016).

Las fuentes extracordilleranas EP1 y HU están disponibles todo el año, son de fácil acceso y ofrecen obsidianas de buena calidad. Sin embargo, a diferencia de las fuentes cordilleranas, no tienen una representación importante en Mendoza hasta los últimos dos mil años (Salgán et al. 2012). ${ }^{5}$

Estudios sobre asignación geoquímica de artefactos arqueológicos en una amplia región del centro de Argentina y Chile han permitido identificar un patrón temporal en el uso: la mayor diversificación de fuentes y las distancias más grandes de desplazamiento tuvieron lugar desde la segunda mitad del Holoceno tardío. En otras regiones de Argentina también se ha observado que los últimos milenios registran la mayor intensidad de uso y circulación de este recurso (Belardi et al. 2006; Pintar et al. 2016; Franco et al. 2017; Stern 2018, entre otros). En el NOA, la fracción temporal entre 2200-400

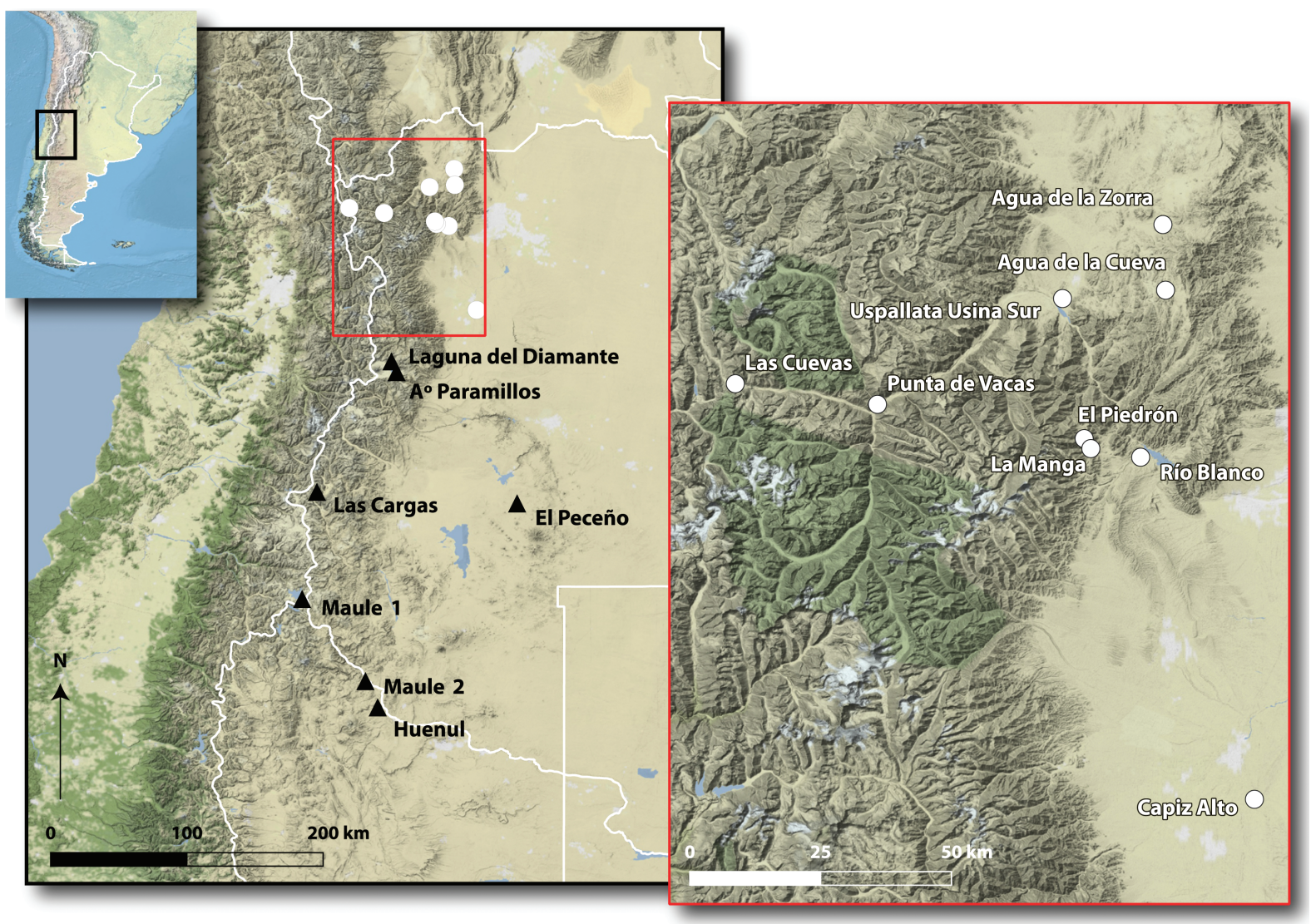

Figura 1. Ubicación de fuentes de obsidiana entre los $34^{\circ}$ y $38^{\circ} \mathrm{S}$ (mapa base: Stamen Terrain Map, http://maps.stamen.com). 
años AP es considerada como lapso de consolidación del contacto de intercambio de larga distancia (Yacobaccio et al. 2004). Esta inflexión temporal, que podría tener impacto en una escala extrarregional, es relevante también para tener en cuenta en la asignación de artefactos de obsidiana en distintos contextos del norte de Mendoza. Hace ca. 2000 años AP se registró un crecimiento demográfico e incorporación de prácticas agrícolas y pastoriles, proceso que incluyó mayor énfasis en el procesamiento de productos silvestres y la continuidad de prácticas de caza (Gasco et al. 2011; Gil et al. 2014; Llano et al. 2017). Estos cambios implicaron el establecimiento de bases semipermanentes en valles intermontanos, circuitos de movilidad más restringidos, sistemas de aprovisionamiento que remiten a áreas o territorios más reducidos y el establecimiento de interacciones de larga distancia (Cortegoso 2014). Estas transformaciones fueron acompañadas por la incorporación de bienes e innovaciones tecnológicas. Algunos indicadores arqueológicos son casas pozo, macrorrestos botánicos de cultígenos, posibles restos óseos de llamas cargueras, estilos cerámicos locales y foráneos (principalmente de Chile central), recursos líticos alóctonos (obsidianas) y la propulsión de flechas por medio de arcos (Frigolé y Gasco 2016; Castro et al. 2018).

Asumiendo que la obsidiana llegó a estos contextos como resultado del desplazamiento por largas distancias, una estimación sobre la variabilidad de las fuentes presentes según rangos cronológicos y tipos artefactuales aporta a la comprensión sobre la complejización económica y social que se está estudiando en las últimas décadas desde múltiples enfoques.

\section{MATERIALES Y MÉTODOS}

\section{Sitios y muestras}

El universo analizado $(\mathrm{N}=61)$ comprende todas las muestras de obsidiana registradas en contextos recuperados por este equipo en el norte de Mendoza y una colección de museo. Corresponden a sitios emplazados en unidades geomorfológicas que ofrecen significativas variaciones altitudinales $y$ cuyos ambientes fueron utilizados de forma complementaria: cordillera $(N=5)$, valles intermontanos: Uspallata y Potrerillos $(\mathrm{N}=2)$; precordillera $(\mathrm{N}=2)$ y planicie oriental $(\mathrm{N}=1)$ (Tabla 1, Figura 1$)$.

La mayor parte de las piezas fueron recuperadas en estratos de aleros y reparos rocosos localizados en cordillera y precordillera, con representaciones que alcanzan un máximo de $0,5 \%$. Es una excepción el ajuar del enterratorio de Uspallata Usina Sur, con 14 puntas talladas en obsidiana. En los valles intermontanos se practicaron grandes excavaciones, particularmente en Potrerillos, donde se estudiaron unidades domésticas en terrazas de ambas márgenes del río Mendoza (Cortegoso et al. 2014). No obstante, estos ambientes muestran muy baja presencia de obsidiana. De igual modo, se han excavado sitios con unidades domésticas y cementerios en planicie, como Capiz Alto y Barrancas (Durán y Novellino 2003; Novellino et al. 2013), donde dicha tendencia es aún más marcada.

Los sitios que contienen la mayor parte de elementos de obsidiana se ubican en ambientes altos (por encima de los $2000 \mathrm{msnm}$ ). Se trata, en general, de aleros y reparos rocosos de uso estacional que evidencian en sus contextos prácticas asociadas a actividades estacionales principalmente vinculadas a la caza. Agua de la Cueva es el sitio con la secuencia ocupacional más extendida, con un registro en el sector sur que inicia en el límite Pleistoceno-Holoceno (García 2003). En el sector norte -de donde se obtuvieron las muestras- hay dos bloques cronoestratigráficos discontinuos, correspondientes, el más temprano, a grupos exclusivamente cazadores y recolectores (ca. 9400-7400 años AP), y el más tardío, a poblaciones con bases residenciales en los valles y con estrategias económicas diversificadas que incluían actividades agropastoriles y extractivas (ca. 2000-1200 años AP) (Frigolé y Gasco 2016; Castro y Yebra 2018).

El Piedrón, en cordillera, tiene un registro de uso recurrente durante el final del Holoceno medio (ca. 5500-4500 años AP). Las actividades de caza y la evidencia de circuitos de movilidad muy amplios caracterizan los contextos recuperados en el sitio y los que contienen la mayor parte del registro de obsidiana (Cortegoso 2005). En la capa superior también se recuperaron elementos de obsidiana; puesto que está alterada y presentaba materiales modernos, no ha sido datada.

Los sitos La Manga Resguardo y La Manga a cielo abierto, muy próximos al alero antes mencionado, se ubican sobre las márgenes de una vega en un área abierta. Este emplazamiento tiene una cronología sincrónica con una de las fases de ocupación del valle de Potrerillos (ca. 1100 años AP) (Marsh y Cortegoso 2014). Los análisis de materiales 
La presencia de obsidiana en contextos arqueológicos del norte de Mendoza: interacciones... Intersecciones en Antropología 20 (2), agosto-diciembre. 2019. ISSN-e 1850-373X

\begin{tabular}{|c|c|c|c|c|c|c|}
\hline \multirow[b]{2}{*}{ Sitio } & \multirow{2}{*}{$\begin{array}{l}\text { Cronología } \\
\left({ }^{14} \mathrm{C}\right)\end{array}$} & \multicolumn{5}{|c|}{ Tipos tecnomorfológicos y procedencia } \\
\hline & & $\begin{array}{l}\text { Fragmento } \\
\text { indif. }\end{array}$ & Lasca & $\begin{array}{l}\text { Micro y ultra- } \\
\text { microlasca }\end{array}$ & Instrumentos & $\begin{array}{l}\text { Lasca de } \\
\text { reactivación }\end{array}$ \\
\hline \multirow[t]{2}{*}{ Las Cuevas (inédito) } & са. $6000 \mathrm{AP}$ & $\mathrm{LC}(\mathrm{N}=1)$ & - & $\mathrm{LC}(\mathrm{N}=1)$ & - & - \\
\hline & са. 1200 AP & $\begin{array}{l}\text { DESC-B } \\
(\mathrm{N}=1)\end{array}$ & - & $\begin{array}{l}\text { DESC-B } \\
(N=10)\end{array}$ & - & - \\
\hline \multirow{3}{*}{$\begin{array}{l}\text { El Piedrón } \\
\text { (Cortegoso 2005) }\end{array}$} & $5460 \pm 130$ & $\mathrm{AP}(\mathrm{N}=2)$ & - & - & - & $\mathrm{LC}(\mathrm{N}=1)$ \\
\hline & $4560 \pm 120$ & $\begin{array}{l}\operatorname{AP}(\mathrm{N}=1) \\
\mathrm{LC}(\mathrm{N}=1)\end{array}$ & - & $\begin{array}{l}\mathrm{AP}(\mathrm{N}=3), \mathrm{LC} \\
(\mathrm{N}=1)\end{array}$ & $\begin{array}{l}\operatorname{AP}(N=2) \\
\operatorname{LM} 1(N=1)\end{array}$ & - \\
\hline & \begin{tabular}{|l|} 
Capa superior \\
(alterado con \\
material moderno)
\end{tabular} & $\mathrm{NA}(\mathrm{N}=1)$ & - & $\mathrm{EP}(\mathrm{N}=4)$ & - & - \\
\hline \multirow[t]{2}{*}{$\begin{array}{l}\text { La Manga Resguardo } \\
\text { (Cortegoso 2008) }\end{array}$} & $\begin{array}{l}1110 \pm 70 \\
1000 \pm 60\end{array}$ & $\mathrm{LC}(\mathrm{N}=1)$ & $\begin{array}{l}\mathrm{LC} \\
(\mathrm{N}=1)\end{array}$ & $\begin{array}{l}\mathrm{LC}(\mathrm{N}=1) \\
\mathrm{AP}(\mathrm{N}=1)\end{array}$ & - & - \\
\hline & $\begin{array}{l}\text { Recolección } \\
\text { superficial }\end{array}$ & - & - & - & $\mathrm{HU}(\mathrm{N}=1)$ & - \\
\hline \multirow{2}{*}{$\begin{array}{l}\text { Quebrada de La Manga } \\
\text { (Cortegoso 2008) }\end{array}$} & $1100 \pm 70$ & $\mathrm{AP}(\mathrm{N}=1)$ & - & - & - & - \\
\hline & $\begin{array}{l}\text { Recolección } \\
\text { superficial }\end{array}$ & - & - & - & LM1 $(\mathrm{N}=1)$ & - \\
\hline Punta de Vacas (inédito) & $\begin{array}{l}\text { Recolección } \\
\text { superficial }\end{array}$ & - & $\begin{array}{l}\mathrm{LC} \\
(\mathrm{N}=1)\end{array}$ & - & - & - \\
\hline \multirow{3}{*}{$\begin{array}{l}\text { Agua de la Cueva sector } \\
\text { Norte } \\
\text { (Lucero et al. 2006; } \\
\text { Castro y Yebra 2018) }\end{array}$} & $7420 \pm 90$ & - & - & $\mathrm{LC}(\mathrm{N}=1)$ & - & - \\
\hline & $1820 \pm 60$ & - & - & $\mathrm{LC}(\mathrm{N}=1)$ & - & - \\
\hline & $\begin{array}{l}1330 \pm 60 \\
1220 \pm 70\end{array}$ & $\begin{array}{l}\text { DESC-B } \\
(\mathrm{N}=1)\end{array}$ & $\begin{array}{l}\text { DESC-B } \\
(N=1)\end{array}$ & $\begin{array}{l}\text { DESC-B } \\
(\mathrm{N}=2)\end{array}$ & - & - \\
\hline $\begin{array}{l}\text { Agua de la Zorra } \\
\text { (Cortegoso et al. 2017) }\end{array}$ & $\begin{array}{l}\text { Recolección } \\
\text { superficial }\end{array}$ & $\mathrm{LD}(\mathrm{N}=1)$ & - & - & - & - \\
\hline $\begin{array}{l}\text { Río Blanco } 01 \\
\text { (Cortegoso 2006) }\end{array}$ & $1220 \pm 80$ & - & - & $\mathrm{LC}(\mathrm{N}=1)$ & - & - \\
\hline $\begin{array}{l}\text { Usina Sur } \\
\text { (Schobinger 1974-1976) }\end{array}$ & $580 \pm 90$ & - & - & - & $\mathrm{LC}(\mathrm{N}=14)$ & - \\
\hline $\begin{array}{l}\text { Capiz Alto } \\
\text { (Durán y Novellino } \\
\text { 2003; Gil et al. 2014) }\end{array}$ & $423 \pm 41$ & - & - & - & LM1 $(\mathrm{N}=1)$ & - \\
\hline
\end{tabular}

Tabla 1. Sitios arqueológicos: cronología, tipos tecnomorfológicos y procedencia de las piezas.

líticos y cerámicos han permitido afirmar que se trataría de ocupaciones estacionales de grupos con economías diversificadas y base residencial en el valle (Cortegoso 2008; Frigolé 2017).

Las Cuevas S8 es un sitio que está en proceso de excavación; próximo al límite internacional, también tiene un registro que se inicia en el Holoceno medio y continúa hasta el tardío. Sus contextos remiten a actividades estacionales vinculadas a la caza.

Pocos elementos de ambientes altos provienen de recolecciones de superficie; una de estas excepciones es un fragmento de talla de obsidiana recuperado en el sito Agua de la Zorra. Se trata de un taller de talla asociado a la explotación de rocas silíceas criptocristalinas del Bosque de Darwin (Cortegoso et al. 2017).

Los valles intermontanos han sido objeto de excavaciones de gran magnitud; sin embargo, como se mencionó, presentan un registro casi nulo de obsidiana. Es una excepción el elemento recuperado en el sitio Río Blanco y que está asociado a la excavación en planta de una unidad doméstica (ca. 1200 años AP). El fechado lo ubica dentro del patrón de ocupación de Potrerillos; varios sitios con casas semisubterráneas han permitido identificar dos fases de ocupaciones dispersas en asentamientos similares y contemporáneos (Cortegoso 2006; Marsh y Cortegoso 2014).

Finalmente, se incluyen en este trabajo dos sitios de carácter funerario. Uspallata Usina Sur es un enterratorio múltiple asociado al inicio del período incaico (ca. 500 años AP) (Marsh et al. 2017; Durán et al. 2018). El sitio originalmente excavado por Schobinger (1974-1976) contenía ofrendas de objetos de metal y de piedra alisada y tallada. El valioso conjunto de puntas de obsidiana intactas que integraban los ajuares ha sido objeto de estudios 
experimentales y geoquímicos previamente publicados (Nami et al. 2015). En la planicie del este de Mendoza se encuentra el sitio Capiz Alto, también con múltiples entierros humanos y contextos del período de contacto que incluyen materiales de origen europeo (ca. 450-400 años AP) (Novellino et al. 2003).

\section{Análisis geoquímicos y tecnológicos}

A excepción de las puntas que integran el ajuar de Usina Sur y la muestra de Capiz Alto, todos los artefactos se analizaron en 2017 mediante fluorescencia de rayos $X$ dispersiva de energía no destructiva (ED-XRF) utilizando un instrumento portátil propiedad del MURR en préstamo a la Universidad de Cuyo en Mendoza. ${ }^{6}$ La asignación de fuentes implicó comparaciones entre los datos de composición para los artefactos y la base de datos de obsidiana MURR XRF y NAA para fuentes en Argentina. Los resultados de asignación para muestras arqueológicas de toda la provincia de Mendoza permiten graficar las señales químicas de las fuentes regionales, como así también dos elipses que agrupan muestras con similitudes químicas (Desconocidas A y B) pero de las cuales se desconoce la localización espacial de la fuente. En este estudio, todas las mediciones de XRF emplearon un espectrómetro de dispersión de energía Bruker Tracer-5i XRF. Cada muestra se analizó durante un minuto para facilitar las mediciones de los siguientes elementos: $\mathrm{K}, \mathrm{Ca}$, $\mathrm{Ti}, \mathrm{Mn}, \mathrm{Fe}, \mathrm{Rb}, \mathrm{Sr}, \mathrm{Y}, \mathrm{Zr}$ y Nb . Sin embargo, para fines de suministro, los elementos más importantes son: Rb, Sr, Y, Zr y Nb. La calibración del Tracer-5i se realizó midiendo un conjunto de 40 muestras de fuentes de obsidiana previamente estudiadas mediante análisis de activación de neutrones (NAA), espectrometría de masa plasmática acoplada inductivamente (ICP-MS) y XRF (Glascock y MacDonald 2018).

El análisis lítico es macroscópico y morfotecnológico (sensu Aschero 1983). Con el objetivo de evaluar actividades de talla desarrolladas en cada espacio se reconstruyen secuencias reductivas, que incluyen las siguientes etapas: adquisición (formas naturales), preparación (núcleos e indiferenciados), talla (lascas externas e internas), formatización (micro-ultramicrolascas), instrumentos (artefactos formatizados y litos modificados por uso) y mantenimiento (lascas de reactivación de filo) (sensu Cortegoso 2008).

\section{RESULTADOS}

\section{Distribución de muestras en el espacio}

A continuación, se presentan las concentraciones para todos los elementos químicos medidos en cada muestra (Tabla 2).

\begin{tabular}{|c|c|c|c|c|c|c|c|}
\hline Muestra & Sitios & Grupo químico & $\mathbf{R b}$ & Sr & $\mathbf{Y}$ & $\mathrm{Zr}$ & $\mathrm{Nb}$ \\
\hline MZN001 & \multirow{6}{*}{ AGC } & LC & 93,5 & 144,0 & 13,3 & 98,7 & 9,0 \\
\hline MZN002 & & \begin{tabular}{|l} 
DESC-B \\
\end{tabular} & 113,9 & 111,6 & 22,3 & 163,2 & 5,9 \\
\hline MZN003 & & \begin{tabular}{|l|} 
DESC-B \\
\end{tabular} & 133,7 & 127,3 & 10,7 & 143,2 & 7,4 \\
\hline MZN004 & & \begin{tabular}{|l} 
DESC-B \\
\end{tabular} & 222,7 & 223,5 & 25,1 & 241,6 & 10,2 \\
\hline MZN005 & & LC & 76,4 & 112,8 & 11,2 & 97,2 & 6,0 \\
\hline MZN006 & & DESC-B & 135,3 & 128,1 & 14,6 & 162,7 & 8,1 \\
\hline MZN007 & \multirow{7}{*}{$\begin{array}{l}\text { La } \\
\text { Manga }\end{array}$} & $\mathrm{HU}$ & 96,7 & 83,2 & 14,0 & 70,8 & 12,8 \\
\hline MZN008 & & \begin{tabular}{|l|}
$\mathrm{LC}$ \\
\end{tabular} & 93,3 & 132,3 & 13,7 & 93,7 & 5,3 \\
\hline MZN009 & & $\mathrm{AP}$ & 103,4 & 186,3 & 18,4 & 109,3 & 11,4 \\
\hline MZN010 & & \begin{tabular}{|l|}
$\mathrm{LC}$ \\
\end{tabular} & 92,9 & 143,5 & 14,7 & 106,6 & 5,5 \\
\hline MZN011 & & \begin{tabular}{|l|}
$\mathrm{LC}$ \\
\end{tabular} & 93,6 & 137,9 & 13,7 & 100,5 & 4,9 \\
\hline MZN012 & & $\mathrm{AP}$ & 105,2 & 201,0 & 18,3 & 119,6 & 14,4 \\
\hline MZN013 & & LM1 & 146,9 & 81,4 & 19,6 & 167,5 & 9,8 \\
\hline MZN014 & $\begin{array}{l}\text { Punta } \\
\text { de Vacas }\end{array}$ & LC & 114,1 & 167,2 & 19,1 & 116,2 & 7,3 \\
\hline MZN015 & \multirow{17}{*}{$\begin{array}{l}\text { El } \\
\text { Piedrón }\end{array}$} & $\mathrm{AP}$ & 106,1 & 191,2 & 18,1 & 112,8 & 14,6 \\
\hline MZN016 & & $\mathrm{AP}$ & 103,7 & 198,4 & 16,8 & 114,0 & 11,1 \\
\hline MZN017 & & AP & 98,2 & 187,9 & 18,2 & 114,5 & 11,8 \\
\hline MZN018 & & LC & 110,4 & 159,4 & 15,3 & 111,7 & 8,0 \\
\hline MZN019 & & \begin{tabular}{|l} 
EP1 \\
\end{tabular} & 200,4 & 207,5 & 6,2 & 105,3 & 24,0 \\
\hline MZN020 & & \begin{tabular}{|l|} 
EP1 \\
\end{tabular} & 240,9 & 234,9 & 7,8 & 108,9 & 25,1 \\
\hline MZN021 & & EP1 & 223,5 & 232,6 & 10,6 & 105,3 & 20,7 \\
\hline MZN022 & & LC & 118,9 & 191,7 & 22,1 & 128,8 & 16,4 \\
\hline MZN023 & & \begin{tabular}{|l|} 
LM1 \\
\end{tabular} & 150,8 & 77,6 & 21,0 & 162,2 & 11,1 \\
\hline MZN024 & & $\mathrm{AP}$ & 91,1 & 166,3 & 15,2 & 95,5 & 11,1 \\
\hline MZN025 & & AP & 91,4 & 176,4 & 15,6 & 100,5 & 11,7 \\
\hline MZN026 & & \begin{tabular}{|l} 
EP1 \\
\end{tabular} & 185,0 & 187,1 & 7,5 & 85,1 & 17,9 \\
\hline MZN027 & & $\overline{\mathrm{AP}}$ & 88,6 & 162,7 & 13,7 & 99,4 & 8,5 \\
\hline MZN028 & & \begin{tabular}{|l|}
$\mathrm{LC}$ \\
\end{tabular} & 136,4 & 175,4 & 16,1 & 122,5 & 5,8 \\
\hline MZN029 & & $\overline{A P}$ & 83,4 & 147,4 & 12,3 & 92,7 & 11,6 \\
\hline MZN030 & & AP & 85,5 & 146,3 & 13,2 & 94,2 & 7,7 \\
\hline MNEP05 & & NA & 72 & 69 & 10 & 45 & 5 \\
\hline MZN032 & $\begin{array}{l}\text { Agua de } \\
\text { la Zorra }\end{array}$ & LD & 134,0 & 48,8 & 17,4 & 71,4 & 10,7 \\
\hline MZN033 & \multirow{13}{*}{$\begin{array}{l}\text { Las } \\
\text { Cuevas }\end{array}$} & DESC-B & 142,9 & 134,9 & 9,3 & 159,8 & 7,3 \\
\hline MZN034 & & \begin{tabular}{|l|} 
DESC-B \\
\end{tabular} & 142,3 & 146,6 & 15,0 & 170,8 & 6,1 \\
\hline MZN035 & & DESC-B & 114,2 & 115,5 & 9,9 & 128,5 & 4,5 \\
\hline MZN036 & & \begin{tabular}{|l|} 
DESC-B \\
\end{tabular} & 135,7 & 133,4 & 11,6 & 154,9 & 4,4 \\
\hline MZN037 & & \begin{tabular}{|l} 
DESC-B \\
\end{tabular} & 126,8 & 127,4 & 11,3 & 141,3 & 5,7 \\
\hline MZN038 & & \begin{tabular}{|l|} 
DESC-B \\
\end{tabular} & 123,2 & 118,1 & 13,6 & 141,3 & 6,7 \\
\hline MZN039 & & \begin{tabular}{|l} 
DESC-B \\
\end{tabular} & 121,8 & 122,9 & 11,1 & 141,0 & 5,4 \\
\hline MZN040 & & \begin{tabular}{|l|} 
DESC-B \\
\end{tabular} & 159,3 & 152,1 & 16,0 & 166,9 & 8,6 \\
\hline MZN041 & & \begin{tabular}{|l|} 
DESC-B \\
\end{tabular} & 130,2 & 124,3 & 12,3 & 144,7 & 6,5 \\
\hline MZN042 & & DESC-B & 124,4 & 132,5 & 11,9 & 146,8 & 7,6 \\
\hline MZN043 & & \begin{tabular}{|l} 
DESC-B \\
\end{tabular} & 123,9 & 128,5 & 9,5 & 142,6 & 6,6 \\
\hline MZN044 & & \begin{tabular}{|l|}
$\mathrm{LC}$ \\
\end{tabular} & 114,1 & 169,5 & 21,3 & 115,2 & 9,3 \\
\hline MZN045 & & LC & 100,0 & 151,3 & 17,5 & 111,7 & 10,4 \\
\hline MZN046 & \begin{tabular}{|l|} 
Río \\
Blanco 01
\end{tabular} & LC & 92,7 & 131,5 & 11,2 & 99,2 & 6,6 \\
\hline C-ALTO-32 & Capiz Alto & LM1 & 137 & 100 & 14 & 154 & 9 \\
\hline \begin{tabular}{|l} 
MUUS01 \\
\end{tabular} & \multirow{14}{*}{$\begin{array}{l}\text { Usina } \\
\text { Sur }\end{array}$} & LC & 105 & 215 & 13 & 121 & 7 \\
\hline MUUSO2 & & LC & 96 & 172 & 11 & 87 & 6 \\
\hline MUUS03 & & \begin{tabular}{|l|}
$\mathrm{LC}$ \\
\end{tabular} & 97 & 168 & 11 & 86 & 5 \\
\hline MUUS04 & & \begin{tabular}{|l|} 
LC \\
\end{tabular} & 98 & 169 & 13 & 82 & 5 \\
\hline MUUS05 & & LC & 100 & 180 & 14 & 92 & 5 \\
\hline MUUS06 & & \begin{tabular}{|l|}
$\mathrm{LC}$ \\
\end{tabular} & 101 & 194 & 13 & 104 & 6 \\
\hline MUUS07 & & \begin{tabular}{|l}
$\mathrm{LC}$ \\
\end{tabular} & 94 & 173 & 13 & 85 & 5 \\
\hline MUUS08 & & LC & 96 & 178 & 12 & 86 & 5 \\
\hline MUUS09 & & \begin{tabular}{|l|}
$\mathrm{LC}$ \\
\end{tabular} & 101 & 181 & 13 & 95 & 5 \\
\hline MUUS10R & & LC & 93 & 171 & 11 & 83 & 5 \\
\hline MUUS11 & & LC & 99 & 187 & 13 & 98 & 6 \\
\hline MUUS12 & & LC & 103 & 184 & 14 & 98 & 5 \\
\hline MUUS13 & & LC & 98 & 177 & 12 & 86 & 6 \\
\hline MUUS14 & & LC & 96 & 171 & 13 & 82 & 5 \\
\hline
\end{tabular}

Nota: NA= No asignada.

Tabla 2. Geoquímica de obsidianas arqueológicas en el norte de Mendoza $\left(32^{\circ}-33^{\circ} \mathrm{S}\right)$. 
Las fuentes se encuentran representadas en las siguientes proporciones: LC 43\% ( $\mathrm{N}=26)$, DESC-B $25 \%(N=15), \operatorname{AP} 16 \%(N=10), \operatorname{EP1} 7 \%(N=4)$, LM1 5\% ( $\mathrm{N}=3)$, HU, LD y NA $2 \%$ cada una $(\mathrm{N}=1 \mathrm{c} / \mathrm{u})$. En la Figura 2 se grafica la distribución de obsidianas arqueológicas según las unidades geomorfológicas del norte provincial.

En las planicies del este, la presencia de obsidiana es muy baja $(\mathrm{N}=1)$. Además de excepcional por la escasísima representación, la muestra recuperada en el sitio Capiz Alto corresponde a la fuente LM1. Esta es la fuente cordillerana más distante del norte de Mendoza (284 km del sitio) y la menos representada en toda la secuencia de ocupación humana del área. Es excepcional también el elemento recuperado en ese sitio de entierro, un punzón entero muy aguzado y completamente retocado (Durán y Novellino 2003).

La columna de los valles intermontanos en gran medida grafica las puntas de proyectil de Usina Sur $(\mathrm{N}=14)$, sitio compuesto casi en su totalidad por objetos votivos procedentes de la vertiente occidental de la cordillera. En el valle de Potrerillos solo se registra un artefacto de obsidiana procedente del sitio Río Blanco. Todos estos elementos han sido asignados a LC.

En precordillera se recuperó obsidiana en los sitios AGC $(N=6)$ y Agua de la Zorra $(N=1)$. En el primer sitio se registraron obsidianas de LC $(\mathrm{N}=2)$ y de la fuente DESC-B $(N=4)$, y en el segundo, un artefacto asignado a LD. Este último puede ser el caso más excepcional del registro regional, ya que esa fuente cordillerana está casi en su totalidad restringida al interior de la caldera del Diamante y no hay otros sitios de Argentina que tengan esta señal, aun cuando se han excavado otros muy próximos

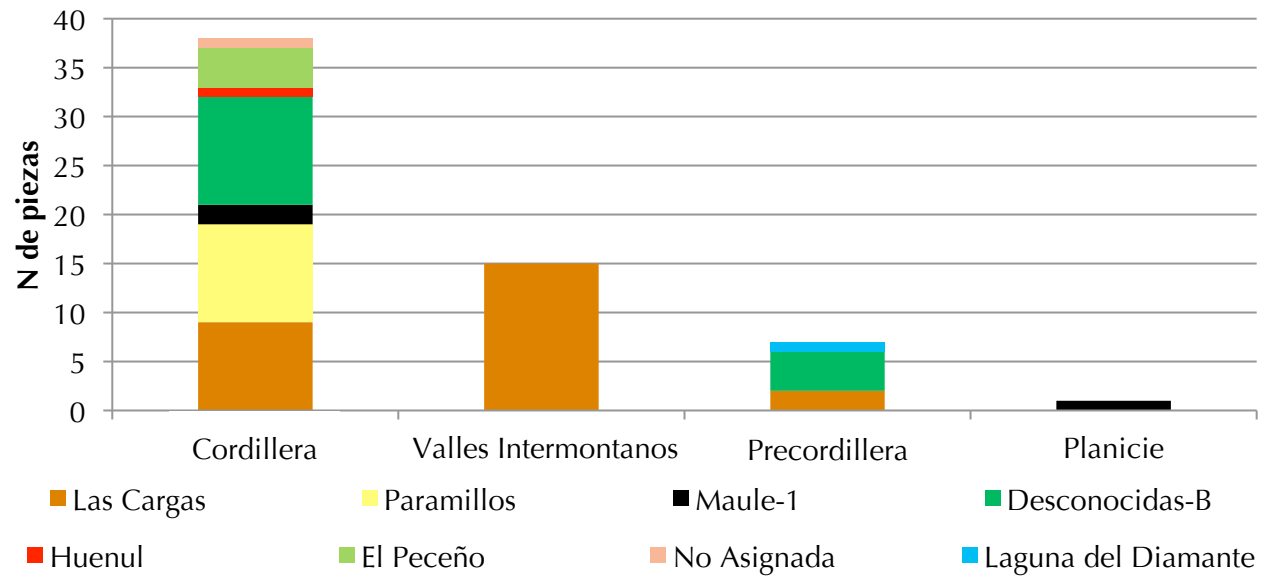

Figura 2. Asignación a fuentes ubicadas entre $34^{\circ}-37^{\circ} \mathrm{S}$ de muestras de obsidiana del norte de Mendoza según unidades geomorfológicas. en la franja sur de las cabeceras del río Diamante (Neme et al. 2016). Solo tiene una muy baja presencia fuera del área de disponibilidad natural en sitios localizados en las cabeceras del río Maipo, en la vertiente occidental andina (Cortegoso et al. 2016).

En cordillera se registra más del $62 \%(\mathrm{~N}=38)$ de las obsidianas. Estas piezas proceden de cinco sitios: Las Cuevas $(N=13)$, El Piedrón $(N=17)$, La Manga Resguardo ( $N=5)$, Quebrada de La Manga $(N=2)$, Punta de Vacas $(N=1)$. Excepto LD, están presentes todas las fuentes cordilleranas y extracordilleranas estudiadas en la macrorregión: $\operatorname{AP}(N=10), \operatorname{LC}(N=9), \operatorname{EP}(n=4), \operatorname{LM} 1(n=2)$ y $\mathrm{HU}(\mathrm{N}=1)$. Incluso, se identificaron piezas de DESC-B $(N=11)$ y $N A(N=1)$. Así, en los sitios de altura aparece la mayor variabilidad de fuentes.

\section{Distribución de muestras en el tiempo}

La dispersión temporal de las muestras (Figura 3) refleja un patrón asimilable al de los pulsos de ocupaciones humanas en la región, con discontinuidades en la señal arqueológica registradas aun en escalas más amplias (Neme y Gil 2009). La obsidiana más temprana (LC) es del Holoceno medio inicial ( $N=1)$, conjunto datado ca. 7400 años AP del sitio AGC. Luego no hay muestras en un rango del Holoceno medio (entre ca. 7000-6000 años AP); esta fracción tiene una señal arqueológica baja y relativamente alternada en ambas laderas andinas (Méndez et al. 2015).

La obsidiana vuelve a estar presente cuando se registran nuevamente ocupaciones humanas ca. 5500 años AP (N = 3) y de manera más significativa hacia el final del Holoceno medio ca. 4500 años $(\mathrm{N}=11)$, con un nuevo pulso que incluye incremento de la fuente AP. En la cuenca del Maipo, en Chile, su explotación se remonta al Holoceno temprano. En el pulso final del Holoceno medio también aparece el registro más temprano de la fuente cordillerana LM1, la más meridional de Mendoza. 


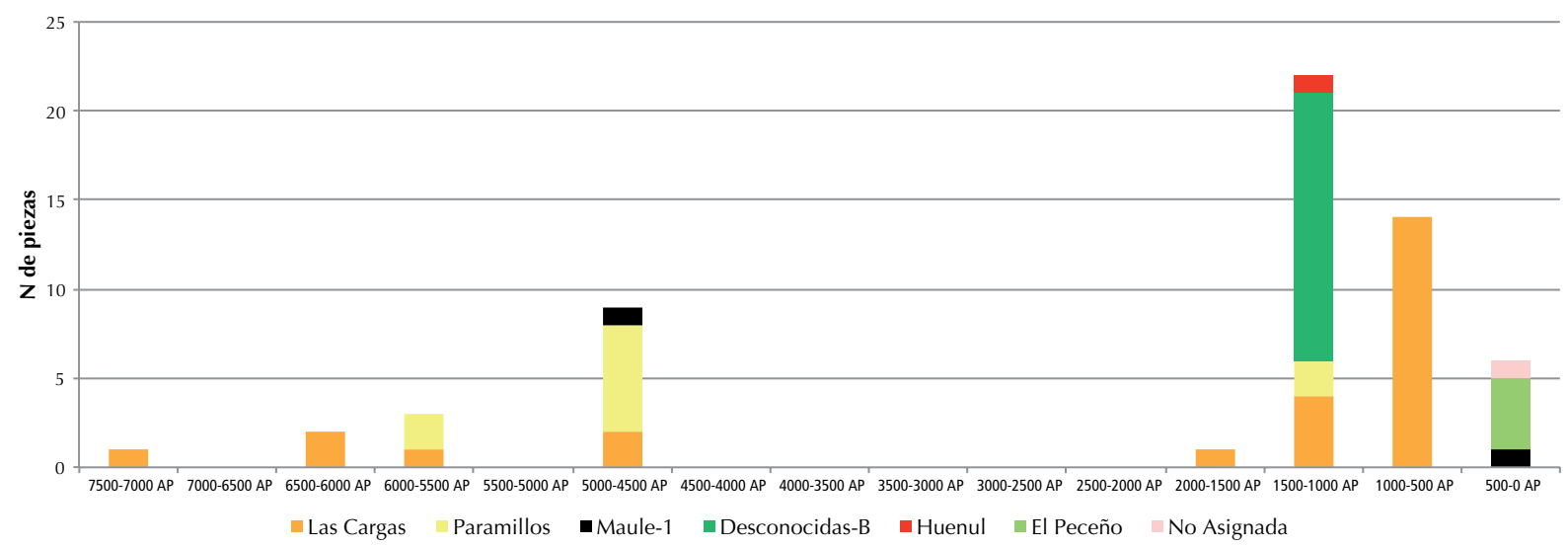

Figura 3. Asignación a fuentes ubicadas entre $34^{\circ}-37^{\circ} \mathrm{S}$ de muestras de obsidiana del norte de Mendoza según rangos temporales.

Después de este lapso, hay una ausencia de registro arqueológico en general y de obsidiana en particular (con una sola muestra ca. $1700 \mathrm{AP}$ ). Entre ca. 1500-1000 años AP se registra un volumen significativo de obsidiana ( $N=22)$, cronología que podría vincularse a ocupaciones humanas efectivas en todos los ambientes del norte de Mendoza. La fuente LC es la más representada en este período en la cuenca del río Blanco, en sitios articulados dentro de circuitos de movilidad anual (Cortegoso 2008). En La Manga Resguardo, cordillera, una pieza fue asignada a AP y el ápice de una punta de proyectil a la fuente extracordillerana HU. Este elemento representa el mayor desplazamiento registrado en sitios de la provincia $(500 \mathrm{~km})$. En este rango temporal se registra la presencia del tipo químico identificado como DESC-B.

El siguiente rango temporal solo tiene registro de LC y corresponde en su totalidad al ajuar de Usina Sur, cuya cronología lo ubica en el límite temporal de la instalación del Imperio en la región (Marsh et al. 2017). El registro más tardío e incluso poscontacto marca una diferencia significativa. En Capiz, un contexto correspondiente al primer siglo de contacto registra la fuente de cordillera más distante LM1. Aparece en esta fracción temporal una señal NA y por primera vez la fuente extracordillerana EP. Esta última se registra en el estrato superficial de El Piedrón, que tiene materiales tardíos alternados con modernos; único estrato que registra acceso a bienes provenientes de un área extracordillerana cuya circulación, aun en el área de influencia de la fuente, no supera los 1000 años AP (Cortegoso et al. 2012).

\section{La expresión tecnológica de obsidianas en el norte de Mendoza}

En cordillera y precordillera existe mayor variabilidad de productos de talla y artefactos (Tabla 1 y Figura 4). La formatización y el mantenimiento de artefactos representan el 57\% de los elementos de obsidiana en la cordillera y el $66 \%$ en la precordillera, esto es significativo en los registros más tempranos. Además, en cordillera, casi el 13\% de la muestra está integrada por fragmentos de artefactos y puntas de proyectil (fracturadas). En los ambientes de altura solo están representadas las etapas finales de las secuencias reductivas de instrumentos, como por ejemplo el mantenimiento y descarte de armas de caza que perdieron su vida útil. Esto denota actividades posiblemente vinculadas al mantenimiento de artefactos en uso que formaban parte de un tool kit transportable (sensu Binford 1980).

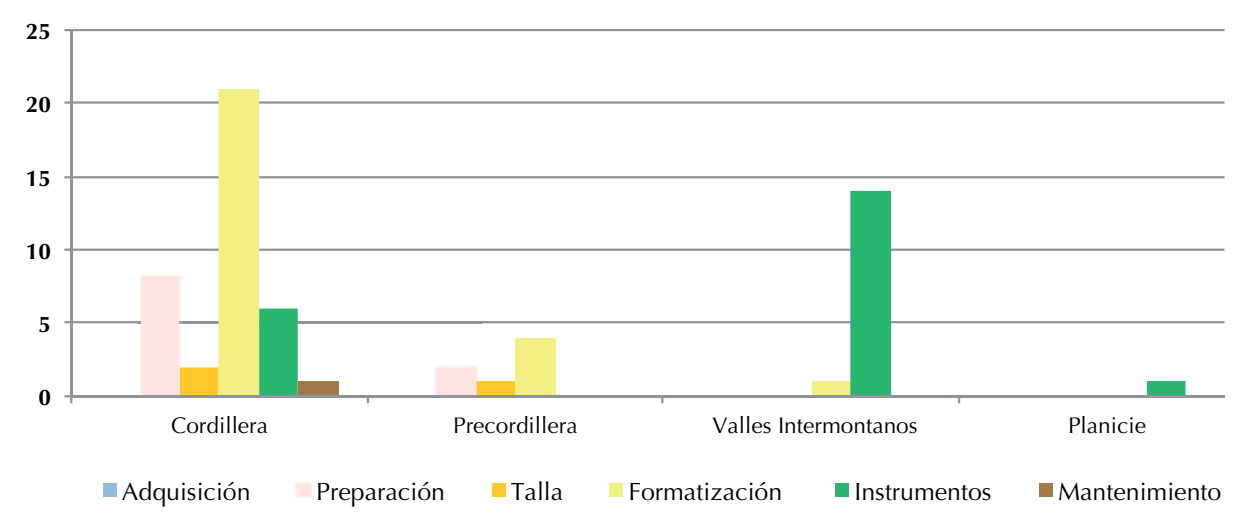

Figura 4. Secuencias reductivas de obsidianas por unidades geomorfológicas. 
En los demás ambientes, por el escaso número o la excepcionalidad de los contextos, no es posible definir secuencias o inferir estrategias de organización y uso (Figura 4). Los objetos más significativos son delicadas piezas enteras, excepcionales por su confección y asociadas a entierros.

\section{DISCUSIÓN}

La obsidiana en el norte de Mendoza está presente desde el Holoceno medio inicial y principalmente en registros de sitios localizados en ambientes de altura. La discontinuidad temporal de presencia/ausencia en el rango cronológico de las muestras analizadas es asimilable a la señal arqueológica en la región. Es decir, existe una relación entre ocupaciones de sitios en ambientes altos y circulación de obsidiana. Las fuentes cordilleranas en general son las que tienen mayor representación; LC no es la más próxima pero aportó el mayor volumen, un elemento que refuerza la existencia de mecanismos de acceso indirecto. Junto con DESC-B, suma la mayor cantidad de elementos exóticos al registro del norte de Mendoza. Teniendo en cuenta este patrón, la segunda señal podría corresponder también a una fuente cordillerana.

La tendencia temporal implica una mayor circulación y diversidad hacia el Holoceno tardío, algo que también se ha registrado en otras áreas de Argentina. En el norte se ha vinculado a la consolidación de intercambio de larga distancia (Yacobaccio et al. 2004); en Patagonia, a una incrementada cantidad de interacción cultural (Stern 2018). Estas diferencias están contrastadas por las estrategias económicas de las sociedades en las que se registran. En el norte de Mendoza, hasta los últimos milenios, la obsidiana está presente en contextos de grupos cazadores y recolectores; luego, en poblaciones con economías diversificadas.

LC aparece de modo relativamente continuo desde ca. 7400 años AP hasta la llegada de los incas. El uso de esta fuente se ha datado in situ desde ca. 10.000 años AP, y existiría una discontinuidad entre 7800-6700 años AP (Salgán et al. 2015). En esta fracción también se han analizado reorganizaciones poblacionales a gran escala a uno y otro lado de los Andes (Méndez et al. 2015). La presencia más temprana en AGC debe interpretarse a la luz de tales fenómenos y en una escala macrorregional (Lucero et al. 2006). El aumento en la frecuencia, tanto como la incorporación de otras fuentes en los contextos posteriores de cazadores y recolectores en cordillera sugieren procesos acumulativos vinculados a flujos de información (sensu Méndez et al. 2012). Las modalidades de interacción de estos grupos no implican necesariamente sistematicidad (Pallo y Borrero 2015) y remiten, en este caso, a un eje principal a lo largo de los Andes.

En los sitios de altura, la obsidiana se habría utilizado con una estrategia de conservación vinculada principalmente al mantenimiento de artefactos de caza; actividad persistente en los registros del norte de Mendoza hasta tiempos tardíos. En el contexto de acceso indirecto, la conservación de instrumentos no es determinada estrictamente por un comportamiento anticipatorio relativo a la organización de la subsistencia, sino por el grado en el cual los instrumentos serán reemplazados a través de los canales de la red de intercambio (McAnany 1988). La prolongación de la vida útil de equipos transportables podría explicar entonces las similitudes tecnológicas de las muestras de obsidiana en ambientes de altura; en otros, por tratarse de hallazgos aislados, no es posible avanzar hacia una caracterización tecnológica detallada.

La continuidad en las prácticas de caza permite explicar las uniformidades tecnológicas de los sitios en ambientes altos en todo el rango cronológico estudiado. En tanto que los cambios en los sistemas de abastecimiento entre sociedades exclusivamente cazadoras recolectoras y las más tardías con bases económicas diversificadas sugieren, para estas últimas, una articulación de formas de interacción y mayor circulación de bienes exóticos. La obsidiana en el norte de Mendoza se hace más recurrente y con mayor diversidad de fuentes en el último milenio, incluyendo las extracordilleranas. En esta fracción temporal, como se señaló en los antecedentes, otros indicadores del registro señalan movilidad reducida y economías que incluyen plantas y animales domésticos. En este contexto, es probable que la presencia de obsidiana refleje -junto con otros bienes foráneos- una sistematización en las vías de interacción. También para regiones trasandinas se ha puesto énfasis en que, hacia fines del Holoceno, el intercambio sistemático de obsidiana habría comenzado en algunas áreas como el centro-sur de Chile (Campbell et al. 2017). Según estos autores, los aspectos estéticos inusuales del recurso y el aumento de la complejidad de las redes sociales desarrolladas en la región después de ca. 1000 cal. AP explicarían la mayor intensidad en este tipo de evidencia. 
El conjunto del ajuar de Usina Sur es excepcional; las puntas de proyectil intactas denotan el valor simbólico de la ofrenda en el marco de una extendida red de circulación de bienes e información que conectaba a sociedades que poblaron distintos ambientes de los Andes centrales argentino-chilenos (Durán y Planella 1989; Lagiglia 2001) y reflejaría una diferenciación y complejidad social sin antecedentes (Schobinger 1974-1976). Sin este conjunto, la fuente LC muestra en volumen una circulación equiparable a la de Arroyo Paramillos. ${ }^{7}$ Las demás señales tienen porcentajes muy bajos.

Los mecanismos que pueden explicar la presencia de obsidiana en contextos arqueológicos del norte de Mendoza pudieron involucrar una gama compleja de situaciones distintas a lo largo del tiempo. Las muestras recuperadas en contextos de sociedades cazadoras-recolectoras podrían indicar mayores desplazamientos y su uso efectivo para el mantenimiento de armas. Sin embargo, la obsidiana aparece con mayor frecuencia en contextos tardíos para los que otros indicadores han permitido establecer que se ocuparon espacios más reducidos y se diversificó la base de la subsistencia. En consecuencia, implicarían el establecimiento y/o consolidación de redes de interacción a mayores distancias. Teniendo en cuenta el origen y las áreas de dispersión de las fuentes utilizadas, estas redes vinculaban de manera troncal poblaciones de ambas vertientes cordilleranas.

La obsidiana es un elemento exótico en el norte de Mendoza; sin embargo, en este trabajo se ha presentado evidencia sobre su integración en sistemas de interacción que se remontan por lo menos a ca. 7000 años AP. Esta materia prima circuló con mayor intensidad y a mayor distancia en los últimos milenios como parte de procesos de regionalización que se han expresado en escalas geográficas muy amplias. Las tendencias que surgen de este trabajo podrán ser de utilidad para enfocar los mecanismos específicos en contextos acotados temporal y espacialmente.

\section{Agradecimientos}

Este trabajo ha sido financiado mediante PIPCONICET 11220150100301 y el PICT-0940 de la ANPCyT. Agradecemos a Erik Marsh, Mike Glascock, Martín Giesso y Ramiro Barberena por su colaboración en estos estudios.

\section{REFERENCIAS CITADAS}

Aschero, C.

1983 Ensayo para una clasificación morfológica de artefactos líticos aplicada a estudios tipológicos comparativos. Informe presentado al Consejo Nacional de Investigaciones Científicas y Técnicas. Apéndices A - C. Revisión. Cátedra de Ergología y Tecnología (FFyLUBA). Buenos Aires. MS.

Barberena, R., V. Durán, P. Novellino, D. Winocur, A. Benítez, A. Tessone, M. Quiroga, E. Marsh, A. Gasco, V. Cortegoso, G. Lucero, C. Llano y K. Knudson

2017 Scale of human mobility in the southern Andes (Argentina and Chile): A new framework based on strontium isotopes. American Journal of Physical Anthropology 164: 305-320.

Barberena, R., M. Fernández, A. Rughini, K. Borrazzo, R. Garvey, G. Lucero, C. Della Negra, G. Romero Villanueva, V. Durán, V. Cortegoso, M. Giesso, C. Klesner, B.

MacDonald y M. Glascock

2018 Deconstructing a Complex Obsidian 'Source-scape': A Geoarchaeological and Geochemical Approach in Northwestern Patagonia. Geoarchaeology 34: 30-41.

Belardi, J. B., P. Tiberi, C. Stern y A. Súnico

2006 Al Este del Cerro Pampa: ampliación del área de disponibilidad de obsidiana de la Pampa del Asador (Provincia de Santa Cruz). Intersecciones en Antropología 7: 27-36.

Binford, L.

1980 Willow Smoke and Dog's Tails: Hunter-Gatherer Settlement Systems and Archaeological Site Formation. American Antiquity 45: 4-20.

Campbell, R., C. Stern y A. Peñaloza

2017 Obsidian in archaeological sites on Mocha Island, southern Chile: Implications of its provenience. Journal of Archaeological Science: Reports 13: 617-624.

Castro, S., L. Yebra, E. Marsh, V. Cortegoso y G. Lucero 2018 The introduction of the bow and arrow in the Argentine Andes (29-34 $\left.{ }^{\circ} \mathrm{S}\right)$ : a preliminary metric approximation. Journal of Lithic Studies 52 (2). En prensa.

Castro, S. y L. Yebra

2018 Ocupación tardía de la precordillera de Mendoza: organización tecnológica en Agua de la Cueva sector norte (ca. 1700-500 años cal. AP). Anales de Arqueología y Etnología 73 (1): 7-40.

Civalero, M. y N. Franco

2003 Early human occupations in Western Santa Cruz Province, Southernmost South America. Quaternary International 109-110: 77-86. 
Cortegoso, V.

2005 Mid-Holocene Hunters in Cordillera: environment, resources and technological strategies. Quaternary International 132: 71-80.

2006 Comunidades agrícolas en el Valle de Potrerillos (NO de Mendoza) durante el Holoceno tardío: organización de la tecnología y vivienda. Intersecciones en Antropología 7: 77-94.

2008 Disponibilidad de recursos líticos en el Noroeste de Mendoza: cambios en la organización tecnológica en la cuenca del río Blanco. Cazadores-Recolectores del Cono Sur 3: 95-112.

2014 Explotación de ambientes cordilleranos y precordilleranos del Centro-Oeste durante el Holoceno: variabilidad espacial y temporal en la organización de la tecnología lítica. En Arqueología de ambientes de altura de Mendoza y San Juan (Argentina), editado por V. Cortegoso, V. Durán y A. Gasco, pp. 19-42. EDIUNC, Mendoza.

Cortegoso, V., G. Neme, M. Giesso, V. Durán y A. Gil $2012 \mathrm{El}$ uso de la obsidiana en el sur de Mendoza. En Paleoecología humana en el sur de Mendoza, editado por G. Neme y A. Gil, pp. 181-211. Sociedad Argentina de Antropología, Buenos Aires.

Cortegoso, V, V. Durán y A. Gasco (Editores) 2014 Arqueología de ambientes de altura de Mendoza y San Juan (Argentina). EDIUNC, Mendoza.

Cortegoso, V., R. Barberena, V. Durán y G. Lucero 2016 Geographic vectors of human mobility in the Andes (34-36 ${ }^{\circ}$ ): comparative analysis of 'minor' obsidian sources. Quaternary International 422: 81-92.

Cortegoso, V., G. Lucero, S. Castro y D. Winocur 2017 Bosques fósiles y tecnología humana: la explotación de materias primas líticas en el bosque de Darwin, Paramillos (Argentina). Latin American Antiquity 18 (3): 317-336.

De Francesco, A., V. Durán, A. Bloise y G. Neme 2006 Caracterización y procedencia de obsidianas de sitios arqueológicos del área natural protegida Laguna del Diamante (Mendoza, Argentina) con metodología no destructiva por fluorescencia de rayos (XRF). Anales de Arqueología y Etnología 61: 53-67.

Durán, V.

2000 Poblaciones indígenas de Malargüe: su arqueología e historia. CEIDER, Mendoza.

2002 Nuevas consideraciones sobre la problemática arqueológica del valle del río Grande (Malargüe, Mendoza). En Entre montañas y desiertos. Arqueología del sur de Mendoza, editado por A. Gil y G. Neme, pp. 87102. Sociedad Argentina de Antropología, Buenos Aires.
Durán, V. y P. Novellino

2003 Vida y muerte en la frontera del Imperio Español. Estudios arqueológicos y bioantropológicos en un cementerio indígena postcontacto del Centro-Oeste de Argentina. Anales de Arqueología y Etnología 54-55: 119-168.

Durán, E. y M. Planella

1989 Consolidación agroalfarera: Zona Central (900 a 1470 d.C.). En Prehistoria de Chile. Desde sus orígenes hasta los albores de la Conquista, editado por J. Hidalgo, V. Schiapacasse, H. Niemeyer, C. Aldunate y I. Solimano, pp. 313-328. Andrés Bello, Santiago de Chile.

Durán, V., M. Giesso, M. Glascock, G. Neme, A. Gil y L. Sanhueza

2004 Estudios de redes de aprovisionamiento y redes de distribución de obsidiana durante el Holoceno Tardío en el sur de Mendoza (Argentina). Estudios Atacameños 28: 25-43.

Durán, V., A. de Francesco, V. Cortegoso, G. Neme, L. Cornejo y M. Bocci

2012 Caracterización y procedencia de obsidianas de sitios arqueológicos del centro-oeste de Argentina y centro de Chile con metodología no destructiva por fluorescencia de rayos $\mathrm{X}$ (XRF). Intersecciones en Antropología 13 (2): 423-437.

Durán, V., V. Cortegoso, R. Barberena, C. Frigolé, P. Novellino, G. Lucero, L. Yebra, A. Gasco, D. Winocur, A. Benítez y K. Knudson

2018 'To and Fro' the southern Andean highlands (Argentina and Chile): Archaeometric insights on geographic vectors of mobility. Journal of Archaeological Science: Reports 18: 668-678.

Fernández, M., R. Barberena, A. Rughini, A. Giesso, V. Cortegoso, V. Durán, G. Romero Villanueva, K. Borrazo, G. Lucero, R. Garvey, B. McDonald y M. Glascock 2017 Obsidian geochemistry, geoarchaeology, and lithic technology in northern Patagonia (Argentina). Journal of Archaeological Science: Reports 13: 372-381.

Franco, N., G. Brook, N. Cirigliano, C. Stern y L. Vetrisano 201717 de Marzo (Santa Cruz, Argentina): a new distal source of Pampa del Asador type black obsidian and its implications for understanding hunter-gatherer behavior in Patagonia. Journal of Archaeological Science: Reports 12: 232-243.

Frigolé, C.

2017 Tecnología cerámica y movilidad en contextos de cambio. Alfarería del primer milenio A.D en la zona cordillerana de Mendoza. Tesis Doctoral inédita. Facultad de Filosofía y Humanidades, Universidad Nacional de Córdoba, Córdoba. 
Frigolé, C. y A. Gasco

2016 Potters and herders at the southern edge of the Andean world: Risk management and mobility in Northwestern Mendoza, Argentina. Quaternary Internacional 422: 152-162.

García, A.

2003 Los primeros pobladores de los Andes Centrales Argentinos. Zeta, Mendoza.

Garvey, R., T. Carpenter, A. Gil, G. Neme y R. Bettinger 2016 Archaeological Age Estimation Based on Obsidian Hydration Data for Two Southern Andean Sources. Chungara 48 (1): 9-23.

Garreaud, R., M. Vuille, R. Compagnucci y J. Marengo 2008 Present-day South America climate.

Palaeogeography, Palaeoclimatology, Palaeoecology 281: 180-195.

Gasco, A., E. Marsh, C. Frigolé, S. Castro, C. Privitera, R. Moyano y L. Yebra

2011 Actividades domésticas durante los siglos III-VIII d.C. en el valle de Potrerillos (San Ignacio-Mendoza). Un acercamiento desde la osteometría y la tecnología cerámica y lítica. Revista del Museo de Antropología 4: 145-160.

Giesso, M, V. Durán, G. Neme, M. D. Glascock, V.

Cortegoso, A. Gil y L. Sanhueza

2011 A Study of Obsidian Source Usage in the Central Andes of Argentina and Chile. Archaeometry 53 (1): $1-21$.

Gil, A., R. Villalba, A. Ugan, V. Cortegoso, G. Neme, C.T. Michieli, P. Novellino y V. Durán

2014 Isotopic evidence on human bone for declining maize consumption during the little ice age in central western Argentina. Journal of Archeological Science 49: 213-227.

Glascock, M y B. MacDonald

2018 Report on the Analysis of Obsidian Artifacts from Archaeological Sites in Mendoza by ED-XRF Analytical Method. Presentado por Archaeometry Laboratory, University of Missouri-Columbia. MS.

Lagiglia, $\mathrm{H}$.

1979 Dinámica cultural en el Centro Oeste Argentino y sus relaciones con áreas aledañas argentinas y chilenas. Actas del VII Congreso Nacional de Arqueología Chilena II. Altos de Vilches: 531-560.

2001 Los orígenes de la agricultura en la Argentina. En Historia argentina prehispánica, t. I, editado por E. Berberián y A. Nielsen, pp. 41-81. Brujas, Córdoba.
Llambías, E., G. Bertotto, C. Risso e I. Hernando 2010 El volcanismo cuaternario en el retroarco de Payenia: una revisión. Revista de la Asociación Geológica Argentina 67 (2): 278-300.

Llano, C., V. Cortegoso y E. Marsh 2017 Producción hortícola a baja escala en el límite continental del desarrollo andino: un aporte desde la arqueobotánica. Darwiniana, nueva serie 5 (2): 109-125.

Lucero, G., V. Cortegoso y S. Castro 2006 Cazadores-Recolectores del Holoceno temprano: explotación de recursos líticos en el sitio Agua de la Cueva Sector Norte. Anales de Arqueología y Etnología 61: 185-216.

Marsh, E. y V. Cortegoso 2014 Refinando la cronología del valle de Potrerillos mediante modelos de Bayes. En Arqueología de Ambiente de Altura de Mendoza y San Juan (Argentina), editado por V. Cortegoso, V. Durán y A. Gasco, pp. 57-79. EDIUNC, Mendoza.

Marsh, E., R. Kidd, D. Ogburn y V. Durán 2017 Dating the expansion of the Inca Empire: Bayesian models from Ecuador y Argentina. Radiocarbon 59: 117-140.

McAnany, P. 1988 The Effects of Lithic Procurement Strategies on Tool Curation and Recycling. Lithic Technology 17 (1): 3-11.

Meltzer, D.

1989 Was stone exchange among eastern North American paleoindians? En Eastern paleoindian lithic resource use, editado por C. Ellis y J. Lothrop, pp. 1139. Westview Press, Boulder.

Méndez, C., C. R. Stern, O. R. Reyes y F. Mena 2012 Early Holocene long-distance obsidian transport in Central-South Patagonia. Chungara 44 (3): 363-375.

Méndez, C., A. Gil, G. Neme, A. Nuevo Delaunay, V.

Cortegoso, C. Huidobro, V. Durán y A. Maldonado 2015 Mid Holocene radiocarbon ages in the Subtropical Andes $\left(29^{\circ}-35^{\circ} \mathrm{S}\right)$, climatic change and implications for human space organization. Quaternary Intenational 356: 15-26.

Nami, H., V. Durán, V. Cortegoso y M. Giesso 2015 Análisis morfológico-experimental y por fluorescencia de Rayos X de las puntas de proyectil de obsidiana de un ajuar del Periodo Agropecuario Tardío del NO de Mendoza, Argentina. Boletín de la Sociedad Chilena de Arqueología 45: 7-37. 
Neme, G. y A. Gil

2009 Human occupation and increasing mid-Holocene aridity: southern Andean perspectives. Current Anthropology 50 (1): 149-163.

Neme, G., N. Sugrañes, L. Salgán, A. Gil, C. Otaola, M. Giardina, C. Morgan y C. Llano

2016 Risco de los indios: Ocupaciones humanas de altura en la cuenca del río Diamante. Relaciones de la Sociedad Argentina de Antropología XLI (1): 101-130.

Novellino, P., G. Barrientos, S. I. Pérez, V. Bernal y M. Béguelin

2003 Morfometría de las poblaciones humanas tardías del sur de Mendoza. Revista Argentina de Antropología Biológica 5(1): 97.

Novellino, P., N. Brachetta Aporta, L. D'Addona, D. Estrella, V. Bernal, S. Devincenzi, V. Cortegoso y V. Durán 2013 Sitios de entierro de la localidad arqueológica Barrancas, Maipú (Mendoza, Argentina). Intersecciones en Antropología 14: 271-277.

Pallo, M. C. y L. Borrero

2015 ¿Intercambio o movilidad?: una evaluación sobre el uso de escalas de análisis espaciales y curvas de declinación en Patagonia centro-meridional (Argentina). Latin American Antiquity 26: 287-303.

Pintar, E., J. Martínez, C. Aschero y M. Glascock 2016 Obsidian use and mobility during the Early and Middle Holocene in the Salt Puna, NW Argentina. Quaternary International 422: 93-108.

Salgán, L., A. Gil y G. Neme

2012 Obsidianas en La Payunia (sur de Mendoza, Argentina): patrones de distribución e implicancias en la ocupación regional. Magallania 40 (1): 263-277.

Salgán, L., R. Garvey, G. Neme, A. Gil, M. Giesso, M. Glascock y V. Durán

2015 Las Cargas: Characterization of a Southern Andean Obsidian Source and Its Prehistoric Use. Geoarchaeology 30: 139-150.

Schobinger, J.

1974-1976 El enterratorio de Uspallata-Usina-Sur (Prov. Mendoza): Estudio de su ajuar funerario. Anales de Arqueología y Etnología 29-31: 67-89.
Seelenfreund, A., C. Rees, R. Bird, G. Bailey, R. Bárcena y V. Durán

1996 Trace element analysis of obsidian sources and artifacts of central Chile (Maule River basin) and western Argentina (Colorado river). Latin American Antiquity 7 (1): 7-20.

Stern, C.

2018 Obsidian sources and distribution in Patagonia, southernmost South America. Quaternary International 468: 190-205.

Yacobaccio, H., P. Escola, F. Pereyra, M. Lazzari, M. Glascock

2004 Quest for ancient routes: obsidian sourcing research in Northwestern Argentina. Journal of Archaeological Science 31 (2): 193-204.

\section{NOTAS}

1.- Entre ambas vertientes se intercambiaban bienes diversos: valvas de moluscos y cuentas de collar, cañas de colihue (Chusquea sp.) de los bosques neuquinos o chilenos (Durán 2000), obsidiana de las nacientes del Maule (Seelenfreund et al. 1996), entre otros.

2.- Las franjas de cordillera que se comparan son: cuenca cordillerana del río Mendoza, en el norte de Mendoza $\left(32^{\circ} \mathrm{S}\right)$, cuenca cordillerana del río Diamante, en el centro de Mendoza $\left(34^{\circ} \mathrm{S}\right)$, y cuenca del río Barrancas, en el norte de Neuquén $\left(36^{\circ} \mathrm{S}\right)$.

3.- El límite entre las áreas norte y sur de Mendoza se ha establecido en el río Diamante $\left(34^{\circ} \mathrm{S}\right)$. A la llegada de los españoles, el río marcaba el límite sur de la expansión incaica y separaba modos de vida agropastoriles (Huarpes-norte) y de caza y recolección (Puelches-sur) (Durán 2002).

4.- El análisis de hidratación sobre productos de talla provenientes de Las Cargas permitió estimar una explotación continua pero con diferencias de intensidad entre 12.0001800 años AP (Garvey et al. 2016).

5.- Estudios recientes han señalado una distribución más amplia que la originalmente estimada para Cerro Huenul (Fernández et al. 2017).

6.- Algunas de las piezas de mayor tamaño fueron analizadas anteriormente con otros equipos portátiles. El aparato utilizado en 2017 es de mayor precisión para XRF y permite la lectura de elementos químicos en especímenes más pequeños (como microlascas). Por ello, todas las piezas fueron procesadas nuevamente con este aparato.

7.- Otro sitio donde se han asignado elementos a Paramillos $(\mathrm{n}=3)$ es El Manzano Histórico (3336'7.27"S) (Durán et al. 2012). 
166 | V. Cortegoso et al. - Intersecciones en Antropología 20 (2), agosto-diciembre. 2019. ISSN-e 1850-373X 